\title{
REVIEW \\ Systematic review of the methodological quality and outcome measures utilized in exercise interventions for adults with spinal cord injury
}

\author{
SR Silverman ${ }^{1,2}$, LA Schertz ${ }^{1,3}$, HK Yuen ${ }^{1,3}$, JD Lowman $^{1,2}$ and CS Bickel ${ }^{1,2}$
}

\begin{abstract}
Study design: Systematic literature review.
Objectives: The primary aims of this study were to evaluate the methodological quality of exercise intervention studies in adults with spinal cord injuries (SCls); and to classify the reported outcome measures according to the International Classification of Functioning, Disability and Health (ICF).

Methods: Electronic searches of PubMed, CINAHL, SPORTDiscus, PsychINFO, Scopus and the Cochrane Center Register of Controlled Trials from 2001 to 2011 were performed. Selected studies were evaluated for methodological quality using the Downs and Black checklist. Outcome measures were extracted and linked to categories of the ICF using standardized linking rules.

Results: Two-hundred forty abstracts were retrieved, 57 studies met eligibility criteria. The mean methodological quality score was $14.7 \pm 3.2$ out of 28 on the Downs and Black checklist. Three-hundred seventy four outcome measures were extracted with 333 concepts linked to 35 second-level ICF categories across the four components.

Conclusion: Studies of exercise interventions for adults with $\mathrm{SCl}$ included in this review are generally low in methodological quality, primarily reporting outcomes related to the Body Functions and Body Structures components of the ICF. It is recommended that studies employ more vigorous methodological designs to reduce bias and confounding, and include outcome measures targeting more categories in the Activities and Participation component so as to reflect the potential benefits of exercise on health and functioning in this population. Spinal Cord (2012) 50, 718-727; doi:10.1038/sc.2012.78; published online 10 July 2012
\end{abstract}

Keywords: spinal cord injury; exercise; physical activity; outcome measures; ICF; methodological quality

\section{INTRODUCTION}

Spinal cord injuries (SCIs) lead to impairment or loss of function below the level of injury. Impairment or loss of function may ultimately lead to activity limitations, restricted participation in a variety of activities and limitations in community involvement due to restricted mobility. ${ }^{1-3}$ Lack of physical activity in adults with SCI has been shown to increase adiposity, alter lipid metabolism and affect cardiovascular function. ${ }^{4-6}$ These changes may increase a person with SCI's risk for mortality as observed in able-bodied adults. ${ }^{7}$ Therefore, increasing levels of physical activity for individuals with SCI is advocated. ${ }^{8}$

Studies have shown increasing physical activity may be beneficial for individuals with SCI. ${ }^{4,9}$ Previous reviews pertaining to exercise and SCI have focused primarily on improvements in physical capacity measures, ${ }^{9-11}$ without relating the outcome measures to the categories and components of the International Classification of Functioning, Disability and Health (ICF)..$^{12}$ A recent review that linked outcome measures to the ICF in people with SCI reported a large number of outcomes were related to 'Body Structures' and 'Body Functions' components. ${ }^{13}$ This particular review evaluated a random sample of any study involving individuals with SCI without specifically addressing exercise interventions. Linking outcome measures from exercise intervention studies to the ICF is helpful, as it provides a knowledge base about which ICF categories, domains and components have been most frequently targeted, and which areas need to be addressed further in future studies.

Another issue related to studies on exercise interventions in adults with SCI is methodological quality. Evaluating methodological quality of studies on exercise interventions not only provides a guide for clinicians and exercise professionals to make informed decisions on adopting the best available evidence in their practice, but also increases researchers' awareness of areas of methodological deficiency. This information is beneficial when designing studies to avoid those pitfalls and improve scientific quality. Although randomized controlled trials (RCTs) are typically viewed as the highest quality of study, ${ }^{14}$ poorly designed RCTs will not provide evidence that is superior to other study designs.

The purpose of this systematic review was to (1) assess the methodological quality of prospective studies on exercise interventions in adults with SCI and (2) classify the reported study outcome measures according to the ICF.

\section{MATERIALS AND METHODS}

This systematic review was conducted in four major steps. First, we performed an extensive search of several commonly used literature databases to locate studies on exercise and SCI, and selected studies that met eligibility criteria.

${ }^{1}$ Program in Rehabilitation Science, University of Alabama at Birmingham, Birmingham, AL, USA; ${ }^{2}$ Department of Physical Therapy, University of Alabama at Birmingham, Birmingham, AL, USA and ${ }^{3}$ Department of Occupational Therapy, University of Alabama at Birmingham, Birmingham, AL, USA

Correspondence: Dr CS Bickel, Department of Physical Therapy, University of Alabama at Birmingham, SHPB 387; 1539 3rd Avenue South, Birmingham, AL 35294-1212, USA E-mail: bickel@uab.edu

Received 9 January 2012; revised and accepted 7 June 2012; published online 10 July 2012 
Second, studies were scored for methodological quality using the Downs and Black checklist. ${ }^{15}$ Third, outcome measures were extracted from each eligibile study. Finally, the concepts within the outcome measures were linked to the categories of the ICF.

\section{Methodological quality and level of evidence}

The Downs and Black checklist was designed to evaluate the methodological quality of both randomized and nonrandomized comparative studies. ${ }^{15}$ The checklist consists of 27 items that address the following methodological components: reporting, external validity, internal validity (bias and confounding) and power. Twenty-six items were rated either as yes $(=1)$ or no/unable to determine $(=0)$, and one item was rated on a 3-point scale ( $y$ es $=2$, partial $=1$, and no $=0$ ). Scores range from 0 to 28 with higher scores indicating a better methodological quality of the study. The following cutpoints have been suggested to categorize studies by quality: excellent (26-28), good (20-25), fair $(15-19)$ and poor $(\leqslant 14) .{ }^{16}$ Adequate psychometric properties of the Downs and Black checklist, such as internal consistency, test-retest reliability, inter-rater reliability and criterion validity, have been reported elsewhere. ${ }^{15}$ The checklist has been ranked in the top six quality assessment tools suitable for use in systematic reviews. ${ }^{17}$

In addition, for studies utilizing the RCT design, ratings were extracted from the Physiotherapy Evidence Database (PEDro) at www.pedro.org.au. ${ }^{18}$ The PEDro scale consists of 11 items with each item awarding one point when the criterion specified in the item is satisfied. The first item is not included to calculate the PEDro score. Scores range from 0 to 10 with higher scores indicating a better methodological quality of the RCT. The following cutpoints were suggested to categorize studies by quality: excellent (9-10), good $(6-8)$, fair $(4-5)$ and poor $(\leqslant 3) .{ }^{19}$ The PEDro scale has demonstrated adequate reliability for use in systematic reviews of exercise intervention RCTs. ${ }^{20}$ Finally, the strength of evidence of the exercise intervention studies was determined using a modified Sackett's levels of evidence (Table 1). ${ }^{21}$ The levels are ranked 1 through 5 and represent the degree of scientific evidence from clinical studies, with 1 being the highest and 5 the lowest level of evidence.

\section{The ICF}

The outcome measures of the exercise intervention studies were classified using the ICF taxonomy. The ICF is organized into four main components: (1) Body Functions, (2) Body Structures, (3) Activities and Participation, and (4) Environmental Factors. ${ }^{12}$ Each of the four components consists of various domains (that is, chapters). Within each domain/chapter, categories and subcategories are denoted by additional digits. ${ }^{22}$ The ICF uses an alphanumeric system to classify each outcome measure. Letters (b-body functions, s-body structures, d-activities and participation and e-environmental factors) represent the four components, followed by a numeric code starting with the chapter number (one digit) indicating the first level, followed by the second level (two digits) and the third and fourth levels (one digit each), which represent an increase in the level of precision in describing the outcome measures. ${ }^{22}$ For example, 'power of the quadriceps' would follow the code for chapter 7 'Neuromusculoskeletal and movement-related functions' of the component 'Body Functions' which is b7 (first level), then 'muscle power functions' is b730 (second level), and finally 'power of muscles in lower half of the body' is b7303 (third level). Outcome measures extracted from the selected studies were linked to categories in the ICF using linking rules established by Cieza et al. ${ }^{23,24}$ Linking rules include the following: all meaningful concepts

\section{Table 1 Modified Sackett's levels of evidence}

\begin{tabular}{ll} 
Level & Study design \\
\hline 1 & RCT (PEDro score $\geqslant 6$ ) \\
2 & RCT (PEDro score $<6$ ), prospective controlled trial, cohort \\
3 & Case control \\
4 & One-group pretest-posttest, case series \\
5 & Case report
\end{tabular}

Abbreviations: PEDro, Physiotherapy Evidence Database; RCT, randomized controlled trials. within each outcome measure are identified, the aim with which the measure used is considered, outcome measure should be linked to the most precise ICF category, and if information is insufficient for making a decision or the concept is not contained within the ICF, the domains are coded $n d$ (not definable) or $n c$ (not coded). ${ }^{23,24}$

\section{Eligibility criteria}

We aimed to include studies that provided original data on the effects of exercise training in adults with SCI. Studies were eligible for inclusion if the independent variable was an exercise intervention lasting at least 4 weeks in duration with a focus other than task-specific training that is typically conducted in clinical rehabilitation settings (that is, outpatient physical therapy). To be included, the exercise intervention had to be described with a specific frequency, duration and mode, including both aerobic and/or strength training. Studies were excluded if the outcome measures of the prescribed intervention were only task-specific, such as studies utilizing bodyweight supported treadmill training (BWSTT) with outcomes only focused on that task (for example, improvements in gait). Additional exclusion criteria were: not employing a prescribed exercise intervention that had a known frequency, duration and mode (for example, physical activity promotion interventions), respiratory muscle training interventions or studies that included participants with common comorbidities of SCI (for example, traumatic brain injury).

\section{Search strategy}

All types and etiologies of SCI were included to provide a comprehensive, unbiased review of the literature. Electronic databases in PubMed, CINAHL, SPORTDiscus, PsychINFO, Scopus and the Cochrane Center Register of Controlled Trials were last searched on 11 October, 2011. Searches were limited to studies published after 2001 (year of publication of the ICF), english language, adult ( $\geqslant 18$ years) and human studies. Search terms included: exercise, physical activity, resistance training, spinal cord injur*, parapleg*, quadripleg* and tetrapleg*.

\section{Data extraction}

Articles were reviewed independently by two authors (SS and LS). First, articles were evaluated for methodological quality using the Downs and Black checklist. ${ }^{15}$ Quality scores for RCTs were extracted from the PEDro. Study designs were determined by the primary reviewer (SS) with agreement from the second reviewer (LS). The intraclass correlation coefficient for Downs and Black methodological quality scores between the two reviewers was 0.90 . When discrepancies in ratings between reviewers were more than one scoring point, discussion between reviewers with consultation from a third party (SB) was sought to resolve the difference.

Second, outcome measures within each article were extracted by the primary reviewer (SS). Outcome measures were linked to the ICF independently by two reviewers (SS and LS) using standard linking rules. ${ }^{23,24}$ We aimed to use the most precise ICF category (that is, the highest level) possible. For example, quadriceps strength would be linked to the ICF category b7303 'power of muscles in lower half of the body' instead of the broader (that is, lower level) category b730 'muscle power functions.' The Kappa coefficient for the agreement between the two reviewers on classifying the ICF codes of the components was 0.79 . Discrepancies in linking outcomes were discussed between reviewers with consultation from a third party (JL) when necessary.

\section{RESULTS}

\section{Study design}

A total of 240 abstracts were retrieved, 57 studies met the eligibility criteria (Figure 1). Study design, intervention type, methodological quality scores and outcome measures of these 57 studies are presented in Table $2 .^{25-81}$ Of the 57 studies, study designs included RCTs $(n=7)$, prospective controlled trials $(n=9)$, one-group pretestposttest design $(n=33)$, case series $(n=4)$ and case reports $(n=4)$. The number of subjects in each study ranged from 1 to 34 $($ median $=10)$. The most common mode of intervention was 


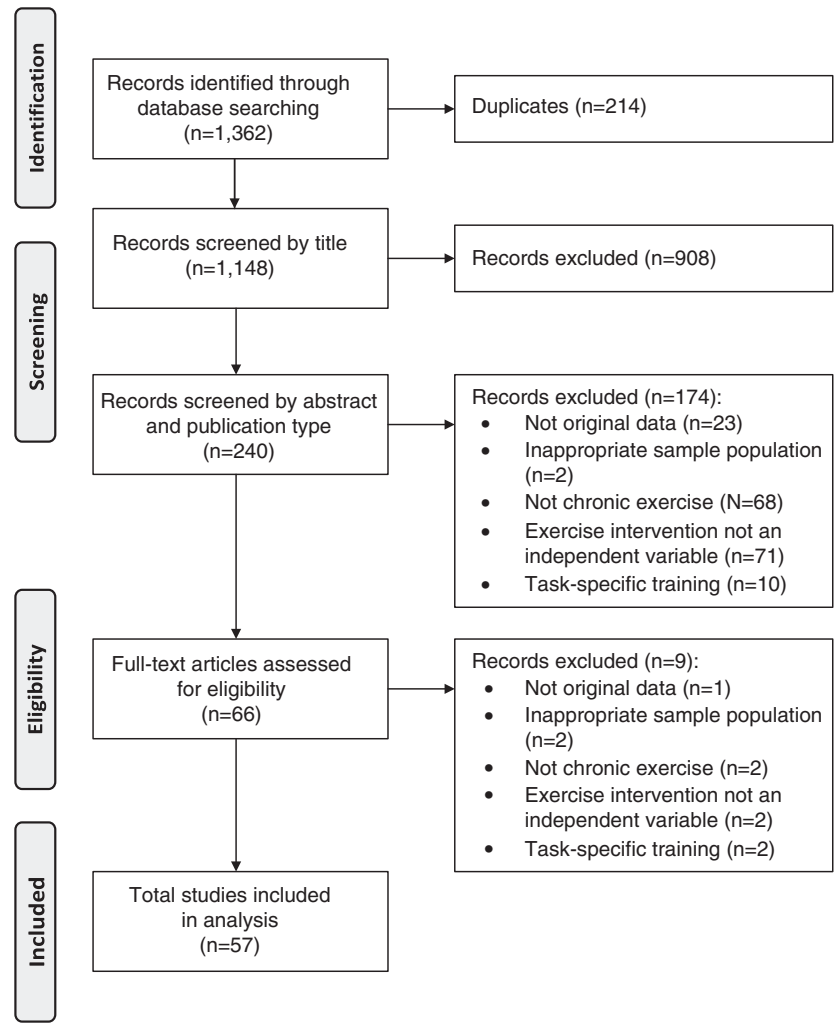

Figure 1 Description of the search strategy.

functional electrical stimulation cycling $(n=13)$, followed by BWSTT $(n=10)$ and functional electrical stimulation resistance training $(n=9)$. Interventions were performed 3-4 days per week with a range of 10-120 min of exercise per session for a mean duration of 18 weeks (s.d. $=18.5$ weeks, three studies were $\geqslant 1$ year duration).

\section{Methodological quality}

The distribution of methodological quality scores based on the Downs and Black checklist are presented in Figure 2. The distribution of scores followed a normal distribution (mean $=14.7$, median $=15) .{ }^{15}$ For the seven RCTs, the PEDro scores ranged from $1-8$, with a median of 5 . Three studies were qualified as level 1 evidence using the modified Sackett's criteria. ${ }^{10}$

\section{Outcome measures linked to the ICF}

A total of 374 outcomes were extracted from the selected studies. For those outcome measures that belong to multi-item surveys, we attempted to retrieve survey documents to include as many ICF codes as possible. Tables 3-5 represent the first and second-level ICF categories with frequency of occurrences in the coded outcome measures. Frequency of occurrence refers to the total number of outcome measures (including survey items) that were linked to a unique ICF code. In 'Body Functions', 5 of 8 first-level categories were addressed, 1 of 7 'Body Structures' categories was addressed, 1 of 9 'Activities and Participation' categories were addressed, and 1 of 5 'Environmental Factors' categories was addressed. There were 41 outcome measures that were not coded to the ICF (listed as not coded, personal factors, or quality of life). Measures that could not be linked to a specific ICF category included quality of life and some anthropometric measures such as waist to hip ratio. Figure 3 represents the percentages of the ICF categories referred to by the outcome measures. A total of 333 concepts could be linked to 35 unique second-level ICF categories.

\section{DISCUSSION}

Of the exercise interventions for people with SCI studies evaluated in this report, the overall methodological quality of the studies was fair according to the suggested categorization scheme for the Downs and Black score. There were very few trials with good methodological quality, or that qualified as level 1 evidence according to the modified Sackett's criteria. Even though exercise is considered to be beneficial for health and is often recommended for people with SCI, this systematic review revealed that there are insufficient numbers of high quality studies of exercise interventions in people with SCI to support the health and function benefits of exercise for this population. In addition, the outcome measures that are typically included in these exercise intervention studies mainly targeted 'Body Functions' without adequately addressing the ICF component of 'Activities and Participation.' It is recommended that the impact of any improvements in body functions and structures from exercise interventions should also be evaluated for their effects on activities and participation.

\section{Study design and methodological quality}

Results of this systematic review give an overview of the current research design and methodological quality issues for studies examining exercise interventions for individuals with SCI. The majority of studies utilized either a one-group pretest-posttest or a nonrandomized controlled design. These findings are in agreement with other reviews of exercise interventions in people with chronic conditions. Two recent reviews of exercise interventions for individuals with traumatic brain injury and muscle disease, yielded only six and three RCTs, respectively. ${ }^{82,83}$ Both reviews concluded that insufficient high quality evidence exists for exercise interventions in these two populations.

Inability to recruit a sufficiently large sample and group heterogeneity in the SCI population may partly explain the frequent use of lower-quality study designs. Given the characteristics of the SCI population, RCTs for this population tend to be small with a high drop-out rate. ${ }^{14}$ This is in agreement with the review by Martin-Ginis and Hicks addressing exercise research issues in this population. ${ }^{14}$ Owing to lack of higher levels of evidence in exercise interventions for people with SCI, current consensus on its efficacy is largely based on well-conducted nonrandomized or single-group studies.

In regard to the scoring of the review studies using the Downs and Black checklist, some studies did not satisfy the criteria because information was not included within the publication. According to the Downs and Black scoring criterion, if the study did not explicitly state a certain requested methodology for a particular item, that item must be scored as not satisfying the criterion. The methodological rating criteria that were most frequently not satisfied in the papers we reviewed were related to blinding, randomization, representativeness of the sample group and adjustment for confounding factors in data analysis. Adequate adjustment for confounding in analysis was a fairly subjective criterion and depended on the rater's opinion of whether or not confounding factors exist. As the SCI population is a heterogeneous group, variables such as lesion level, completeness and age should be controlled for statistically, particularly if the study design did not employ randomization or group matching.

The Downs and Black checklist includes a criterion concerning appropriate statistical tests, ${ }^{15}$ however it is unclear if there was adequate control for the risk of type I errors among the reviewed 
Table 2 Study design and description of relevant studies that met inclusion criteria

\begin{tabular}{|c|c|c|c|c|c|c|c|c|}
\hline \multirow[t]{2}{*}{ References } & \multirow{2}{*}{$\begin{array}{l}\text { Intervention } \\
\text { type }\end{array}$} & \multicolumn{3}{|c|}{ Quality scores } & \multirow{2}{*}{$\begin{array}{l}\text { Study } \\
\text { type }\end{array}$} & \multicolumn{3}{|c|}{ Outcome measures } \\
\hline & & $D$ and $B$ & PEDro & Level & & Improvement $^{\mathrm{a}}$ & Decline & No change \\
\hline Ashe $^{25}$ & FES cycling & 9 & - & 4 & $\begin{array}{l}\text { C. } \\
\text { series }\end{array}$ & BMD, $\%$ of lean, $\%$ of fat & - & - \\
\hline Bizzarini26 & $\begin{array}{l}\text { UE erg and } \\
\text { resistance } \\
\text { training }\end{array}$ & 12 & - & 4 & $\begin{array}{l}\text { Pre/ } \\
\text { Post }\end{array}$ & FES cycle workload & - & $\begin{array}{l}\text { Weight, body composition }{ }^{\mathrm{b}} \text {, testoster- } \\
\text { one/cortisol ratio, CPK level }\end{array}$ \\
\hline Bjerkefors $^{27}$ & $\begin{array}{l}\text { Kayak } \\
\text { ergometer }\end{array}$ & 17 & - & 4 & $\begin{array}{l}\text { Pre/ } \\
\text { Post }\end{array}$ & $\begin{array}{l}\text { Transfer height, WC propulsion, platform mount } \\
\text { height }\end{array}$ & - & WC agility \\
\hline Bjerkefors ${ }^{28}$ & $\begin{array}{l}\text { Kayak } \\
\text { ergometer }\end{array}$ & 20 & - & 4 & $\begin{array}{l}\text { Pre/ } \\
\text { Post }\end{array}$ & Multidirectional shoulder strength ${ }^{b}$ & - & - \\
\hline Bjerkefors ${ }^{29}$ & $\begin{array}{l}\text { Kayak } \\
\text { ergometer }\end{array}$ & 19 & - & 4 & $\begin{array}{l}\text { Pre/ } \\
\text { Post }\end{array}$ & Trunk displacement during perturbation & - & - \\
\hline Bogie $^{30}$ & $\begin{array}{l}\text { Implanted } \\
\text { NMES }\end{array}$ & 15 & - & 4 & $\begin{array}{l}\text { Pre/ } \\
\text { Post }\end{array}$ & Ischial pressure & - & Total interface pressure \\
\hline Bougenot ${ }^{31}$ & $\begin{array}{l}\text { Wheelchair } \\
\text { ergometry }\end{array}$ & 14 & - & 4 & $\begin{array}{l}\text { Pre/ } \\
\text { Post }\end{array}$ & $\mathrm{VO}_{2}, \mathrm{VCO}_{2}$, tolerated power, $\mathrm{SpO}_{2}$ & - & HR, peak ventilation, tidal volume \\
\hline Carvalho 32 & $\mathrm{FES}+\mathrm{BWSTT}$ & 11 & - & 2 & PCT & Muscle CSA (quadriceps) & - & - \\
\hline Carvalho 33 & $\mathrm{FES}+\mathrm{BWSTT}$ & 14 & - & 2 & PCT & Bone biochemical markers & - & BMD (lumbar, femur) \\
\hline Chen $^{34}$ & FES cycling & 13 & - & 2 & PCT & BMD (femur, tibia) & - & BMD (calcaneus) \\
\hline Clark $^{35}$ & $\begin{array}{l}\text { FES knee } \\
\text { extension, } \\
\text { ankle } \\
\text { dorsiflexion }\end{array}$ & 20 & - & 2 & PCT & Body composition & BMD (total) & BMD (hip, spine) \\
\hline Coupaud ${ }^{36}$ & BWSTT & 13 & - & 5 & $\begin{array}{l}\text { C. } \\
\text { report }\end{array}$ & $\begin{array}{l}\text { Bone mineral content, BMD (total, trabecular), } \\
\text { muscle CSA (quadriceps) }\end{array}$ & $\begin{array}{l}\text { Fat CSA } \\
\text { (quadriceps) }\end{array}$ & Bone CSA (femur) \\
\hline Crameri $^{37}$ & FES cycling & 12 & - & 4 & $\begin{array}{l}\text { Pre/ } \\
\text { Post }\end{array}$ & $\begin{array}{l}\text { FES cycle workload, muscle fiber type }{ }^{\text {b }} \\
\text { muscle CSA (quadriceps), muscle capillarization } \\
\text { (quadriceps), citrate synthase level, hexokinase } \\
\text { level }\end{array}$ & - & - \\
\hline de Groot $^{38}$ & UE erg & 14 & 5 & 2 & $\mathrm{RCT}$ & $\mathrm{VO}_{2}$, Power output, blood lipids ${ }^{\mathrm{b}}$ & - & Insulin sensitivity \\
\hline De Mello 39 & $\begin{array}{l}\text { Aerobic } \\
\text { exercise }\end{array}$ & 11 & - & 4 & $\begin{array}{l}\text { Pre/ } \\
\text { Post }\end{array}$ & Leg movements during sleep & - & - \\
\hline Dolbow 40 & UE erg & 9 & - & 5 & $\begin{array}{l}\text { C. } \\
\text { report }\end{array}$ & $\mathrm{VO}_{2}, \%$ body fat & - & BMD (total) \\
\hline $\begin{array}{l}\text { Dyson- } \\
\text { Hudson } 41\end{array}$ & UE erg & 22 & - & 2 & PCT & WUSPI score ${ }^{b}$ & - & - \\
\hline Effing $^{42}$ & BWSTT & 15 & - & 4 & $\begin{array}{l}\text { Pre/ } \\
\text { Post }\end{array}$ & Case series- varied by subject & & \\
\hline El-Sayed ${ }^{43}$ & UE erg & 16 & - & 2 & PCT & $\mathrm{VO}_{2}$, ventilation max, workload & - & Blood lipids ${ }^{b}$ \\
\hline Gerrits $^{44}$ & $\begin{array}{l}\text { FES knee } \\
\text { extension }\end{array}$ & 11 & - & 4 & $\begin{array}{l}\text { C. } \\
\text { series }\end{array}$ & Mean blood velocity, systolic inflow volume & - & $\begin{array}{l}\text { Peak blood velocity, Minimum blood } \\
\text { velocity, End-diastolic velocity }\end{array}$ \\
\hline Gerrits $^{45}$ & FES cycling & 15 & - & 4 & $\begin{array}{l}\text { Pre/ } \\
\text { Post }\end{array}$ & FES force, fatigue resistance & - & $\begin{array}{l}\text { Contraction/relaxation speed } \\
\text { (quadriceps) }\end{array}$ \\
\hline Gerrits 46 & $\begin{array}{l}\text { FES knee } \\
\text { extension, } \\
\text { ankle } \\
\text { dorsiflexion }\end{array}$ & 14 & - & 4 & $\begin{array}{l}\text { Pre/ } \\
\text { Post }\end{array}$ & $\begin{array}{l}\text { Knee extension. strength, Fatigue resistance } \\
\text { (quadriceps), succinate dehydrogenase } \\
\text { (quadriceps) }\end{array}$ & - & $\begin{array}{l}\text { Myosin type I, muscle fiber diameter } \\
\text { (quadriceps), alpha-glycerophosphate } \\
\text { dehydrogenase (quadriceps) }\end{array}$ \\
\hline Giangregorio 47 & BWSTT & 13 & - & 4 & $\begin{array}{l}\text { C. } \\
\text { series }\end{array}$ & $\begin{array}{l}\text { Walking duration, walking speed, BWS, \% lean } \\
\text { mass, muscle CSA (quadriceps) }\end{array}$ & $\begin{array}{l}\text { BMD } \\
\text { (femur, } \\
\text { tibial, } \\
\text { lumbar) }\end{array}$ & Bone biochemical markers \\
\hline Giangregorio 48 & BWSTT & 17 & - & 4 & $\begin{array}{l}\text { Pre/ } \\
\text { Post }\end{array}$ & $\begin{array}{l}\text { Walking duration, walking speed, BWS, body } \\
\text { composition, muscle CSA (quadriceps) }\end{array}$ & BMD (total) & $\begin{array}{l}\text { BMD (spine, femur, tibia), bone bio- } \\
\text { chemical markers }\end{array}$ \\
\hline Gorgey 49 & $\begin{array}{l}\text { NMES + } \\
\text { resistance } \\
\text { knee flex/ } \\
\text { extension }\end{array}$ & 9 & - & 5 & $\begin{array}{l}\text { C. } \\
\text { report }\end{array}$ & $\begin{array}{l}\text { Muscle CSA }{ }^{b}, \text { IM fat, subcutaneous fat, NMES } \\
\text { resistance }\end{array}$ & - & - \\
\hline
\end{tabular}


Table 2 (Continued)

\begin{tabular}{|c|c|c|c|c|c|c|c|c|}
\hline \multirow[t]{2}{*}{ References } & \multirow{2}{*}{$\begin{array}{l}\text { Intervention } \\
\text { type }\end{array}$} & \multicolumn{3}{|c|}{ Quality scores } & \multirow{2}{*}{$\begin{array}{l}\text { Study } \\
\text { type }\end{array}$} & \multicolumn{3}{|c|}{ Outcome measures } \\
\hline & & $D$ and $B$ & PEDro & Level & & Improvement $^{\mathrm{a}}$ & Decline & No change \\
\hline Gregory 50 & $\begin{array}{l}\text { LE resistance } \\
\text { and plyo- } \\
\text { metric } \\
\text { training }\end{array}$ & 10 & - & 4 & $\begin{array}{l}\text { C. } \\
\text { series }\end{array}$ & $\begin{array}{l}\text { Torque (knee extension, plantarflexion), Rate of } \\
\text { torque development (knee extension, plantarflex- } \\
\text { ion), activation deficit (knee extension, } \\
\text { plantarflexion) }\end{array}$ & - & - \\
\hline Griffin $^{51}$ & FES cycling & 16 & - & 4 & $\begin{array}{l}\text { Pre/ } \\
\text { Post }\end{array}$ & Ride time, power, body composition, OGTT, AIS ${ }^{b}$ & - & Cholesterol \\
\hline Grigorenko ${ }^{52}$ & Sea kayaking & 15 & - & 2 & PCT & Acceleration of change (sagittal) & - & $\begin{array}{l}\text { COP change }{ }^{b} \text {, velocity of change }{ }^{b}, \\
\text { acceleration of change (frontal) }\end{array}$ \\
\hline Harvey ${ }^{53}$ & $\begin{array}{l}\text { FES + PRT } \\
\text { on single } \\
\text { quadriceps }\end{array}$ & 20 & 8 & 1 & $\mathrm{RCT}$ & $\begin{array}{l}\text { Quadriceps strength (voluntary), perception of } \\
\text { exercise effectiveness }\end{array}$ & - & $\begin{array}{l}\text { Quadriceps strength (stim), quadriceps } \\
\text { endurance (voluntary, stim), perceived } \\
\text { ability, satisfaction w/ program }\end{array}$ \\
\hline Heesterbeek $^{54}$ & $\begin{array}{l}\text { Hybrid FES } \\
\text { cycling + UE } \\
\text { erg }\end{array}$ & 16 & - & 4 & $\begin{array}{l}\text { Pre/ } \\
\text { Post }\end{array}$ & Leg volume, $\mathrm{VO}_{2}$ & - & $\begin{array}{l}\text { Peak ventilation, peak HR, power } \\
\text { output }\end{array}$ \\
\hline Hicks ${ }^{55}$ & $\begin{array}{l}\text { PRE upper } \\
\text { extremity, UE } \\
\text { erg }\end{array}$ & 20 & 5 & 1 & $\mathrm{RCT}$ & $\begin{array}{l}\text { Walking score, BWS, speed, distance, life satis- } \\
\text { faction, satisfaction w/ physical function }\end{array}$ & - & $\begin{array}{l}\text { Depression, perceived health, perceived } \\
\text { ability for IADL }\end{array}$ \\
\hline Hicks ${ }^{56}$ & BWSTT & 16 & - & 4 & $\begin{array}{l}\text { Pre/ } \\
\text { Post }\end{array}$ & $\begin{array}{l}\text { Peak HR, BP, UE strength }{ }^{b} \text {, power output, satis- } \\
\text { faction w/ physical function, satisfaction w/ } \\
\text { appearance, pain, stress, depression, PQOL, per- } \\
\text { ceived health }\end{array}$ & - & - \\
\hline Jacobs $^{57}$ & $\begin{array}{l}\text { Circuit resis- } \\
\text { tance training }\end{array}$ & 13 & - & 4 & $\begin{array}{l}\text { Pre/ } \\
\text { Post }\end{array}$ & Fatigue resistance, $\mathrm{VO}_{2}$, UE strength ${ }^{\mathrm{b}}$ & - & Elbow flexion/extension strength \\
\hline Jacobs $^{58}$ & $\begin{array}{l}\text { Endurance or } \\
\text { resistance }\end{array}$ & 17 & - & 4 & $\begin{array}{l}\text { Pre/ } \\
\text { Post }\end{array}$ & $\mathrm{VO}_{2}$, Power, UE strength ${ }^{\mathrm{b}}$ & - & Peak ventilation, peak HR, peak RPE \\
\hline$J^{e o n}{ }^{59}$ & FES cycling & 15 & - & 4 & $\begin{array}{l}\text { Pre/ } \\
\text { Post }\end{array}$ & OGTT & - & Insulin secretion, Insulin sensitivity \\
\hline$J^{e o n}{ }^{60}$ & FES rowing & 15 & - & 4 & $\begin{array}{l}\text { Pre/ } \\
\text { Post }\end{array}$ & Fasting glucose, fasting leptin & - & $\begin{array}{l}\text { Body composition, fasting insulin, } \\
\text { insulin resistance }\end{array}$ \\
\hline Kakebeeke 61 & FES cycling & 10 & - & 5 & $\begin{array}{l}\text { C. } \\
\text { report }\end{array}$ & $\begin{array}{l}\mathrm{BMD} \text { (femur), thigh CSA, Fat CSA (thigh, lower } \\
\text { leg), peak } \mathrm{HR}, \mathrm{VO}_{2} \text {, blood lactate }\end{array}$ & - & Muscle CSA (lower leg) \\
\hline Kern ${ }^{62}$ & $\begin{array}{l}\text { FES knee } \\
\text { extension/ } \\
\text { flexion }\end{array}$ & 18 & - & 4 & $\begin{array}{l}\text { Pre/ } \\
\text { Post }\end{array}$ & $\begin{array}{l}\text { Knee extension torque, muscle composition } \\
\text { (quadriceps) }\end{array}$ & - & - \\
\hline Kjaer $^{63}$ & FES cycling & 11 & - & 4 & $\begin{array}{l}\text { Pre/ } \\
\text { Post }\end{array}$ & $\begin{array}{l}\text { Quadriceps: hexokinase, lactate dehydrogenase, } \\
\text { citrate synthase, hydroxyacyl-3-dehydrogenase }\end{array}$ & - & - \\
\hline Latimer ${ }^{64}$ & $\begin{array}{l}\text { PRE UE erg } \\
\text { and resis- } \\
\text { tance training }\end{array}$ & 14 & 2 & 2 & $\mathrm{RCT}$ & $\begin{array}{l}\text { Exercise influences depression through stress, } \\
\text { independent of pain. }\end{array}$ & & \\
\hline Latimer 65 & $\begin{array}{l}\text { PRE UE erg } \\
\text { and resis- } \\
\text { tance training }\end{array}$ & 18 & 1 & 2 & RCT & Stress, depression, PQOL & - & - \\
\hline $\operatorname{Liu}^{66}$ & FES cycling & 15 & - & 4 & $\begin{array}{l}\text { Pre/ } \\
\text { Post }\end{array}$ & $\begin{array}{l}\text { Thigh girth, body composition, knee flex/extension } \\
\text { torque }\end{array}$ & - & - \\
\hline Mahoney 67 & $\begin{array}{l}\text { FES knee } \\
\text { extension }\end{array}$ & 14 & - & 4 & $\begin{array}{l}\text { Pre/ } \\
\text { Post }\end{array}$ & CSA (quadriceps) & - & OGTT, fasting insulin \\
\hline Martin-Ginis ${ }^{68}$ & $\begin{array}{l}\text { UE erg and } \\
\text { resistance } \\
\text { training }\end{array}$ & 22 & 6 & 1 & RCT & $\begin{array}{l}\text { Pain, stress, self-efficacy, perceived } \\
\text { control, PQOL, depression, satisfaction } \\
\text { w/ physical function, satisfaction w/ appearance }\end{array}$ & - & - \\
\hline Mohr69 & FES cycling & 11 & - & 4 & $\begin{array}{l}\text { Pre/ } \\
\text { Post }\end{array}$ & $\begin{array}{l}\text { GLUT-4 transport level, Insulin-stimulated glucose } \\
\text { uptake }\end{array}$ & - & OGTT \\
\hline Nash70 & $\begin{array}{l}\text { circuit resis- } \\
\text { tance training }\end{array}$ & 15 & - & 4 & $\begin{array}{l}\text { Pre/ } \\
\text { Post }\end{array}$ & $\begin{array}{l}\text { UE force, } \mathrm{VO}_{2} \text { peak, anaerobic power, WUSPI } \\
\text { score }^{\mathrm{b}}\end{array}$ & - & - \\
\hline Phillips 71 & BWSTT & 13 & - & 4 & $\begin{array}{l}\text { Pre/ } \\
\text { Post }\end{array}$ & $\begin{array}{l}\text { BWS, gait velocity, glucose tolerance, insulin } \\
\text { response, substrate oxidation, GLUT-4 level, } \\
\text { muscle hexokinase, muscle metabolites }\end{array}$ & - & Body composition \\
\hline
\end{tabular}


Table 2 (Continued)

\begin{tabular}{|c|c|c|c|c|c|c|c|c|}
\hline \multirow[t]{2}{*}{ References } & \multirow{2}{*}{$\begin{array}{l}\text { Intervention } \\
\text { type }\end{array}$} & \multicolumn{3}{|c|}{ Quality scores } & \multirow{2}{*}{$\begin{array}{l}\text { Study } \\
\text { type }\end{array}$} & \multicolumn{3}{|c|}{ Outcome measures } \\
\hline & & $D$ and $B$ & PEDro & Level & & Improvement ${ }^{\mathrm{a}}$ & Decline & No change \\
\hline Sk $\square \mathrm{Id}^{72}$ & FES cycling & 13 & 2 & 2 & $\mathrm{RCT}$ & LE muscle volume & - & $\begin{array}{l}\text { Spasticity }{ }^{\mathrm{b}} \text {, body composition, body } \\
\text { weight }\end{array}$ \\
\hline Soyupek ${ }^{73}$ & BWSTT & 14 & - & 4 & $\begin{array}{l}\text { Pre/ } \\
\text { Post }\end{array}$ & Resting HR, FVC, inspiratory capacity, depression & - & $\begin{array}{l}\text { BP, Maximum inspiratory pressure, } \\
\text { FEV1, FEV1/FVC, expiratory reserve } \\
\text { volume, forced expiratory flow, slow } \\
\text { vital capacity }\end{array}$ \\
\hline Stewart $^{74}$ & BWSTT & 14 & - & 4 & $\begin{array}{l}\text { Pre/ } \\
\text { Post }\end{array}$ & $\begin{array}{l}\text { Ambulatory capacity, cholesterol, muscle fiber } \\
\text { CSA (VL), muscle fiber composition }(V L)^{b} \text {, citrate } \\
\text { synthase }(V L)\end{array}$ & - & - \\
\hline Stoner ${ }^{75}$ & $\begin{array}{l}\text { FES knee } \\
\text { extension }\end{array}$ & 16 & - & 4 & $\begin{array}{l}\text { Pre/ } \\
\text { Post }\end{array}$ & Flow-mediated dilation (posterior tibial artery) & - & $\begin{array}{l}\text { Artery vessel diameter (posterior tibial } \\
\text { artery) }\end{array}$ \\
\hline Sutbeyaz $^{76}$ & $\begin{array}{l}\text { Breathing } \\
\text { exercises and } \\
\text { UE erg }\end{array}$ & 13 & - & 4 & $\begin{array}{l}\text { Pre/ } \\
\text { Post }\end{array}$ & $\begin{array}{l}\mathrm{VO}_{2} \text {, Peak } \mathrm{HR}, \mathrm{HR} \text { reserve, minute ventilation, } \\
\mathrm{SpO}_{2} \text {, power output (UE), exercise time }\end{array}$ & - & $\mathrm{BP}$ \\
\hline Thijssen 77 & $\begin{array}{l}\text { FES cycling \& } \\
\text { UE erg }\end{array}$ & 13 & - & 4 & $\begin{array}{l}\text { Pre/ } \\
\text { Post }\end{array}$ & $\begin{array}{l}\text { VO2, thigh bloodflow, vessel diameter, vascular } \\
\text { resistance, thigh volume, fatigue resistance }\end{array}$ & - & $\begin{array}{l}\text { Forearm bloodflow, calf bloodflow, } \\
\text { mean wall shear weight, forearm cir- } \\
\text { cumference, Lower leg volume }\end{array}$ \\
\hline Valent $^{78}$ & Hand cycling & 19 & - & 4 & $\begin{array}{l}\text { Pre/ } \\
\text { Post }\end{array}$ & $\begin{array}{l}\text { Power output (UE), } \mathrm{VO}_{2} \text {, shoulder abduction } \\
\text { strength }\end{array}$ & - & $\begin{array}{l}\mathrm{RER} \text {, peak } \mathrm{HR}, \mathrm{SpO}_{2} \text {, elbow flexion/ } \\
\text { extension strength, shoulder IR } \\
\text { strength, } \mathrm{FVC} \text {, peak expiratory flow }\end{array}$ \\
\hline Valent $^{79}$ & Hand cycling & 17 & - & 2 & PCT & $\begin{array}{l}\text { Power output (UE), shoulder strength }{ }^{\text {b }} \text {, elbow } \\
\text { flexion strength }\end{array}$ & - & $\begin{array}{l}\text { Peak } \mathrm{HR} \text {, resting } \mathrm{HR}, \mathrm{VO}_{2}, \mathrm{FVC} \text {, } \\
\text { shoulder flexion/extension strength, } \\
\text { elbow extension strength, wheelchair } \\
\text { capacity }\end{array}$ \\
\hline $\begin{array}{l}\text { Van } \\
\text { Duijnhoven } \\
80\end{array}$ & FES cycling & 16 & - & 2 & PCT & - & - & $\begin{array}{l}\text { Malondialdehyde level, superoxide dis- } \\
\text { mutase level, glutathione peroxidase } \\
\text { level }\end{array}$ \\
\hline Wheeler 81 & FES rowing & 16 & - & 4 & $\begin{array}{l}\text { Pre/ } \\
\text { Post }\end{array}$ & $\mathrm{VO}_{2}, \mathrm{SpO}_{2}$ & - & ventilation volume, RER, peak HR \\
\hline
\end{tabular}

Abbreviations: AIS, ASIA impairment score; BMD, bone mineral density; BMI, body mass index; BP, blood pressure; BWS, body weight support; BWSTT, body weight supported treadmill training; COP, center of pressure; CPK, creatine phosphokinase; C. report, case report; C. series, case series; CSA, cross-sectional area; D and B, Downs and Black; FES, functional electrical stimulation; FEV, forced expiratory volume; FVC, forced vital capacity; GLUT, glucose transporter; HR, heart rate; LE, lower extremity; NMES, neuromuscular electrical stimulation; OGTT, oral glucose tolerance test; PCT, prospective controlled trial; PEDro, Physiotherapy Evidence Database; PQOL, perceived quality of life; PRE, progressive resistance exercise; Pre/Post, one group pretest-posttest design; PRT, progressive resistance training; RCT, randomzied controlled trial; RER, respiratory exchange ratio; RPE, rating of perceived exertion; $\mathrm{SpO}_{2}$, oxygen pulse; UE erg, upper extremity ergometry; VL, vastus lateralis; WC, wheelchair; WUSPI, wheelchair user's shoulder pain index.

almprovement indicates physiologically desirable change.

bMultiple items included within measure.

studies. Some studies included a large number of outcome measures, particularly that measuring multidirectional upper-extremity strength; however, the statistical analyses did not always indicate if appropriate statistical adjustments were utilized. Owing to the inherent risk of type I error, researchers should be cautious when considering the inclusion of a high number of outcome measures that address the same research question.

\section{Outcome measures linked to the ICF}

The most commonly addressed outcomes were related to 'muscle power functions' (b730). Within this second-level category, however, muscular strength and power are considered together. This was problematic for outcome measures in exercise intervention studies because power and strength are often considered as different concepts, whereas in the ICF, they share the same code. In addition, 'power of muscles in lower half of the body' is a third-level code; however, there is no corresponding code for power of muscles in upper half of the body. As a result, only the second-level code was used to address upper-extremity strength.
We occasionally had difficulty determining the usefulness of the code 'fatiguability' (b4554) due to the overlapped concept of muscle function when the outcome measure was specific to skeletal muscle fatigue. To overcome this difficulty we chose to code muscle fatigue to 'muscle endurance functions' (b740) and general fatigue to 'fatiguability' (b4554). Another example was 'respiration functions' (b440) that is closely related to exercise tolerance but was utilized for multiple outcomes concerning items such as tidal volume and forced vital capacity.

'General metabolic functions' (b540) was also frequently addressed in the published reports. For the studies examined, this code typically included the outcomes of glucose tolerance as measured by oral glucose tolerance test and blood lipids. We also coded cholesterol and triglyceride levels within this category as they are a component of fat metabolism. When considering components of metabolic syndrome, the b540 category is a useful descriptor because it covers both glucose tolerance and blood lipid levels.

'Body Structures' component was commonly coded within the extracted outcome measures. The most frequent occurrence was 


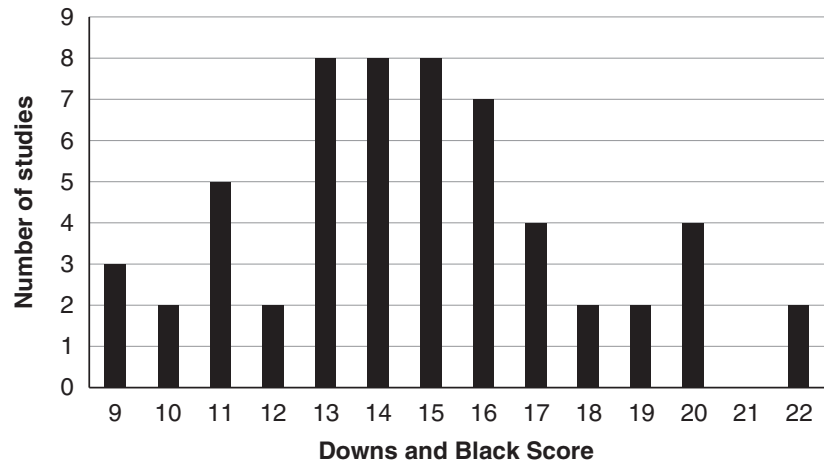

Figure 2 Methodological quality score distribution based on the Downs and Black checklist. Possible range of scores $0-28$.

Table 3 Frequency of second-level categories of the ICF relative to body functions

\begin{tabular}{crc}
\hline ICF code & Description & Frequenc \\
\hline $\begin{array}{c}\text { Chapter 1: Mental functions (12) } \\
\text { b134 }\end{array}$ Sleep functions & 2 \\
b152 & Emotional functions & 10 \\
& & \\
Chapter 2: Sensory functions and pain (5) & 2 \\
b270 & Sensory functions related to temperature and other stimuli & 3 \\
b280 & Pain & \\
& & \\
Chapter 4: Functions of the cardiovascular, hematological, immunological and \\
respiratory systems (81)
\end{tabular}

Chapter 5: Functions of the digestive, metabolic and endocrine systems (50) b530 Weight maintenance functions 15 b540 General metabolic functions 16

b545 Water, mineral, and electrolyte balance functions 9

b555 Endocrine gland functions

Chapter 7: Neuromusculoskeletal and movement-related functions (84)

b730 Muscle power functions

b735 Muscle tone functions

b740 Muscle endurance functions

b750 Motor reflex functions

b755 Involuntary movement reaction functions

b760 Control of voluntary movement functions

Abbreviation: ICF, International Classification of Functioning, Disability and Health.

'structure of the lower extremity' (s750). Within the s750 code we included muscle size, muscle fiber size and muscle cross-sectional area. We chose to use this category (s750) for bone mineral density (BMD) when a specific lower extremity site was stated as an outcome measure. When considering total BMD, following standard linking rules by Cieza et al. ${ }^{23,24}$ the lower-level code s7 was used. However, total BMD may also refer to the ICF 'Body Functions' component of 'maintaining mineral balance' (b5451).
Table 4 Frequency of second-level categories of the ICF relative to body structures

\begin{tabular}{clc} 
ICF code & Description & Frequency \\
\hline $\begin{array}{cl}\text { Chapter } 7: \text { Structures related to movement (54) } \\
\text { s730 }\end{array}$ & Structure of the upper extremity & 1 \\
s740 & Structure of the pelvic region & 1 \\
s750 & Structure of the lower extremity & 45 \\
s760 & Structure of the trunk & 4 \\
s770 & Additional musculoskeletal structures related to movement & 3
\end{tabular}

Table 5 Frequency of second-level categories of the ICF relative to activities and participation and environmental factors

\begin{tabular}{|c|c|c|}
\hline ICF code & Description & Frequency \\
\hline \multicolumn{3}{|c|}{ Activities and Participation } \\
\hline \multicolumn{3}{|c|}{ Chapter 4: Mobility (33) } \\
\hline d410 & Changing basic body position & 1 \\
\hline d420 & Transferring oneself & 7 \\
\hline d430 & Lifting and carrying objects & 4 \\
\hline d450 & Walking & 11 \\
\hline d465 & Moving around using equipment & 8 \\
\hline d475 & Driving & 2 \\
\hline \multicolumn{3}{|c|}{ Chapter 5: Self-care (8) } \\
\hline d510 & Washing oneself & 2 \\
\hline d540 & Dressing & 6 \\
\hline \multicolumn{3}{|c|}{ Chapter 6: Domestic life (2) } \\
\hline d640 & Doing housework & 2 \\
\hline \multicolumn{3}{|c|}{ Environmental Factors } \\
\hline \multicolumn{3}{|c|}{ Chapter 1: Products and technology (4) } \\
\hline e120 & $\begin{array}{l}\text { Products and technology for personal indoor } \\
\text { and outdoor mobility and transportation }\end{array}$ & 4 \\
\hline
\end{tabular}

Abbreviation: ICF, International Classification of Functioning, Disability and Health.

The most frequent code within the 'Activities and Participation' component was $\mathrm{d} 450$ 'walking.' This outcome measure may be overrepresented due to the high number of studies using BWSTT. In studies where the exercise intervention was BWSTT, the outcome measure walking time was frequently used. Examining walking in light of tolerated walking time during BWSTT is coded under the 'Activities and Participation,' however, as an outcome measure during BWSTT, walking could be better suited to the 'gait pattern functions' (b770) category of the 'Body Functions.' Within the 'Activities and Participation' there is also a code for 'mobility'(d4). This includes using assistive devices such as a wheelchair or scooter. Under the category of 'mobility' is $\mathrm{d} 465$ 'moving around using equipment.' This second-level code might be a better representation of mobility from an activities and participation standpoint than 'walking' (d450) among people with chronic conditions such as SCI, because the important concept is the ability to move around in one's environment, not necessarily the ability to walk.

The ICF component 'Activities and Participation' replaces the International Classification of Impairments, Disabilities and Handicaps concept of handicap. ${ }^{84}$ The important difference between the two models is that 'Activities and Participation' refers to an individual's ability to fulfill a role within their specific life situation. ${ }^{84}$ Handicap is 


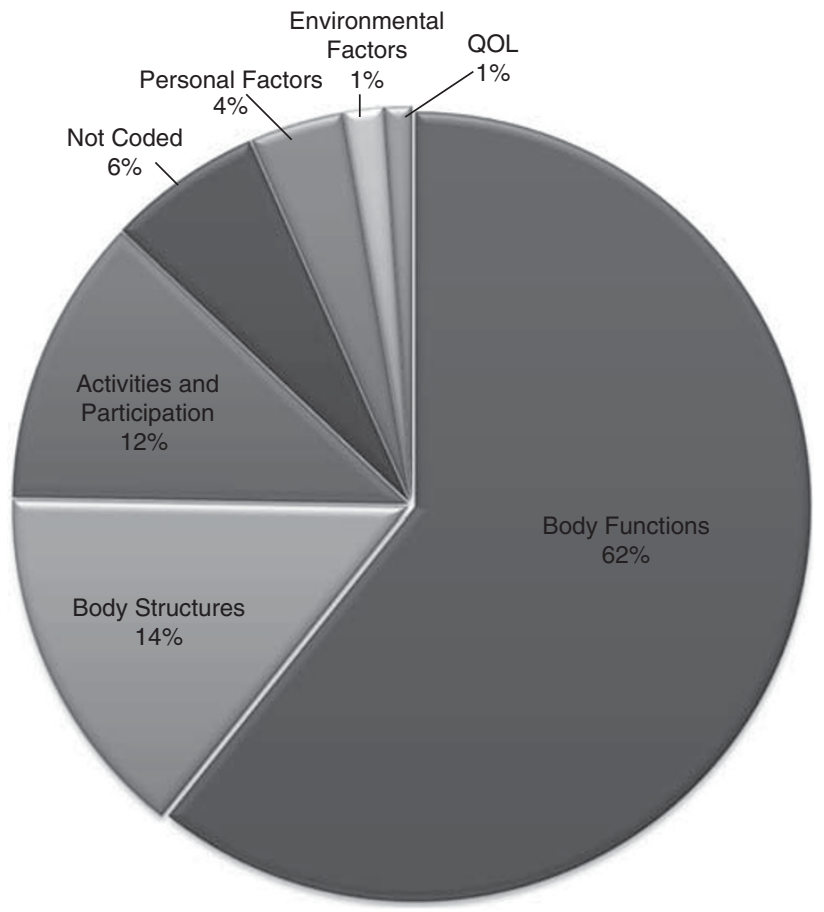

Figure 3 Percentage of outcome measures linked to specific ICF categories. A total of 374 outcomes were extracted.

referred more specifically to fulfillment of roles as defined by society. ${ }^{84}$ Specific instruments for measuring participation in wheelchair users have been developed within the past 10 years. ${ }^{85,86}$ Previous reviews have found multiple validated and reliable instruments that can be used to assess participation by wheelchair users. ${ }^{1,85,86}$ The results of the current review indicate that the concept of participation is still not being well addressed as an outcome of exercise interventions for individuals with SCI, despite the availability of these instruments.

The 'Environmental Factors' component includes social support, assistive technology and aspects of the natural environment. Within the 57 studies evaluated for this review, we extracted only one secondlevel category within the 'Environmental Factors' 'products and technology for personal indoor and outdoor mobility and transportation' (e120). When considering the effects of an exercise intervention, some aspects of the 'Activities and Participation' will be affected by one's environment such as climate and the accessibility and availability of appropriate equipment; however, these were not addressed as an outcome measure by the studies evaluated in this review.

Outcome measures that fell into the categories of 'not defined,' 'not coded' or 'Personal Factors,' component such as quality of life and self-efficacy are currently recognized as weaknesses within the ICF. Some outcome measures that fell into the category of 'not coded' could potentially be incorporated in the existing taxonomy but currently there is not an adequate fit. For example, muscle biochemistry, which involves both body structures and functions, but there are no specific codes for muscle biochemistry within either component.

\section{Limitations}

The studies included in the current review were selected from papers published over a 10-year period from 2001 to 2011. The ICF was introduced in 2001, bringing increased awareness and attention to researchers and scientists that encouraged inclusion of outcome measures that assessed domains other than body structures and functions. ${ }^{87,88}$ Noreau and Shephard ${ }^{89}$ also indicated in their review of studies on exercise after SCI that studies were heavily weighted toward body structures and functions, although they did not use current ICF language. We acknowledge that there were significant contributions to the literature on exercise and SCI before 2001, but including exercise papers before this time would certainly have biased our findings even more as there were few studies with outcome measures that targeted domains other than body structures and functions. ${ }^{89}$

\section{IMPLICATIONS}

The results of this review indicate that studies focusing on exercise interventions for individuals with SCI face both methodological and measurement deficiency. The methodological quality of the studies for this review was generally low. Previous research has suggested that nonrandomized study designs are more frequently conducted due to inherent difficulties in studying chronic condition populations, such as SCI. ${ }^{14}$ Although some evidence for efficacy of exercise interventions for people with SCI has been gleaned from this review, it is necessary for researchers to improve the methodological quality of studies to definitively determine efficacy of the interventions.

Methodological quality can be improved in future studies that utilize nonrandomized controlled designs by improving statistical control for confounding factors such as lesion level and level of completeness. Given that small sample size and heterogeneous characteristics of the participants are always an issue to conducting exercise intervention research in this population, higher methodological quality studies could be achieved by using multi-site RCTs that would allow for a greater number of participants to be recruited. High drop-out rates were another main issue that reduced the methodological quality of the reviewed studies. Future exercise intervention studies for this population may benefit from improving the research design and developing sophisticated incentive strategies to improve participant retention.

Using the ICF as a reference, the results of this review indicate that the majority of outcome measures are classified under the components of 'Body Functions' and 'Body Structures', with 'Environmental Factors' being the least frequently addressed. We believe that future research may benefit from evaluating the influence of environmental factors related to exercise interventions for individuals with SCI. The most frequently addressed category in the 'Activities and Participation' was walking; however, this may have been overrepresented due to a relatively high proportion of reviewed studies $(n=10,17.5 \%)$ using BWSTT. Within the ICF, walking is coded under the 'Activities and Participation' component; however, for individuals with SCI, walking as an outcome measure may be more accurately coded under the 'Body Functions' component if walking was conducted in a controlled setting such as on a treadmill. Whereas, walking in a natural environment such as in one's home or the community may more accurately reflect participation.

Measurement instruments are available to assess participation for people with SCI; however, to our knowledge, they have not been widely implemented as an outcome measure for exercise interventions for people with SCI. Currently available evidence indicates that outcome measures of exercise intervention for individuals with SCI are primarily targeted at body functions and body structures. Future research on the efficacy of exercise interventions on individuals with SCI should place increased emphasis on the outcome measures related to activities and participation, and attempt to include the influence of environment. 


\section{CONFLICT OF INTEREST}

The authors declare no conflict of interest.

\section{ACKNOWLEDGEMENTS}

We would like to thank Susan Smith for her assistance with the search strategy used in this review.

1 Noreau L, Fougeyrollas P, Post M, Asano M. Participation after spinal cord injury: the evolution of conceptualization and measurement. J Neurol Phys Ther 2005; 29 $147-156$.

2 Bucholz AC, McGillivray CF, Pencharz PB. Physical activity levels are low in free-living adults with chronic paraplegia. Obesity 2003; 11: 563-570.

3 Winnick JP. Adapted Physical Activity and Sport, 5th edn. Human Kinetics: Champaign, IL, 2010.

4 Janssen TW, van Oers C, Kamp GJ, TenVoorde BJ, van der Woude LH, Hollander AP. Coronary heart disease risk indicators, aerobic power and physical activity in men with spinal cord injuries. Arch Phys Med Rehabil 1997; 78: 697-705.

5 Bauman WA, Spungen AM. Carbohydrate and lipid metabolism in chronic spinal cord injury. J Spinal Cord Med 2001; 24: 266-277.

6 Fernhall B, Heffernan K, Jae SY, Hedrick AW. Health implications of physical activity in individuals with spinal cord injury: a literature review. J Health Hum Serv Adm 2008, 30: 468-502

7 Schoenborn CA, Stommel M. Adherence to the 2008 adult physical activity guidelines and mortality risk. Am J Preventative Med 2011; 40: 514-521.

8 Whiteneck G, Meade MA, Dijikers M, Tate DG, Bushnik T, Forchheimer MB. Environmental factors and their role in participation and life satisfaction after spinal cord injury. Arch Phys Med Rehabil 2004; 85: 1793-1803.

9 Devillard X, Rimaud D, Roche F, Calmels P. Effects of training programs for spinal cord injury. Annales de Readaptation et de Medicine Physique 2007; 50: 490-498.

10 Sheel WA, Reid WD, Townson AF, Ayas NT, Konnyu KJ. Effects of exercise training and inspiratory muscle training in spinal cord injury: A systematic review. J Spinal Cord Med 2008; 31: 500-508.

11 Valent L, Dallmeijer A, Houdijk H, Talsma E, van der Woude L. The effects of upper body exercise on the physical capacity of people with a spinal cord injury. Clin Rehabil 2007; 21: 315-330.

12 World Health Organization. International Classification of Functioning, Disability and Health. World Health Organization: Geneva, 2001.

13 Post M, Kirchberger I, Scheuringer M, Wollaars M, Geyh S. Outcome parameters in spinal cord injury research: a systematic review using the International Classification of Functioning, Disability and Health (ICF) as a reference. Spinal Cord 2010; 48 522-528.

14 Martin-Ginis KA, Hicks AL. Exercise research issues in the spinal cord injured population. Exerc Sport Sci Rev 2005; 33: 49-53.

15 Downs SH, Black N. The feasibility of creating a checklist for the assessment of the methodological quality both of randomised and non-randomised studies of health care interventions. J Epidemiol Community Health 1998; 52: 377-384.

16 Hooper P, Jutai JW, Strong G, Russell-Minda E. Age-related macular degeneration and low-vision rehabilitation: a systematic review. Can J Opthamol 2008; 43: 180-187.

17 Deeks JJ, Dinnes J, D'Amico R, Sowden AJ, Sakarovitch C, Song F et al. Evaluating non-randomised intervention studies. Health Technol Assess 2003; 7: 1-179.

18 Moseley AM, Herbert RD, Sherrington C, Maher CG. Evidence for physiotherapy practice: a survey of the Physiotherapy Evidence Database (PEDro). Aust J Physiother 2002; 48: 43-49.

19 Teasell RW, Hsieh TJ, Aubut JA, Eng JJ, Krassioukov A, Tu L. Venous thromboembolism after spinal cord injury. Arch Phys Med Rehabil 2009; 90: 232.

20 Maher CG, Sherrington C, Herbert RD, Moseley AM, Elkins M. Reliability of the PEDro scale for rating quality of randomized controlled trials. Phys Ther 2003; 83: 713-721.

21 Sackett DL, Strauss SE, Richardson WS, Rosenberg W, Haynes RB. Evidence-Based Medicine: How to Practice and Teach EBM. Churchill Livingstone: Toronto, Ontario, Canada, 2000.

22 Xiong T, Hartley S. Challenges in linking health-status outcome measures and clinical assessment tools to the ICF. Adv Physiother 2008; 10: 152-156.

23 Cieza A, Borckow T, Ewert T, Amman E, Kollertis B, Chatteriji S et al. Linking healthstatus measurements to the International Classification of Functioning, Disability and Health. J Rehabil Med 2002; 34: 205-210.

24 Cieza A, Geyh S, Chatterji S, Kostanjsek N, Stun B, Stucki G. ICF linking rules: an update based on lessons learned. J Rehabil Med 2005; 37: 212-218.

25 Ashe MC, Eng JJ, Krassioukov AV, Warburton DER, Hung C, Tawashy A. Response to functional electrical stimulation cycling in women with spinal cord injuries using dualenergy X-ray absorptiometry and peripheral quantitative computed tomography: a case series. J Spinal Cord Med 2010; 33: 68-72.

26 Bizzarini E, Saccavini M, Lipanje F, Magrin P, Malisan C, Zampa A. Exercise prescription in subjects with spinal cord injuries. Arch Phys Med Rehabil 2005; 86: 1170-1175.

27 Bjerkefors A, Thorstensson A. Effects of kayak ergometer training on motor perfor mance in paraplegics. Int J Sports Med 2006; 27: 824-829.

28 Bjerkefors A, Jansson A, Thorstensson A. Shoulder muscle strength in paraplegics before and after kayak ergometer training. Eur J Appl Physiol 2006; 97: 613-618.
29 Bjerkefors A, Carpenter MG, Thorstensson A. Dynamic trunk stability is improved in paraplegics following kayak ergometer training. Scand J Med Sci Sports 2007; 17 672-679.

30 Bogie KM, Triolo RJ. Effects of regular use of neuromuscular electrical stimulation on tissue health. J Rehabil Res Dev 2003; 40: 469-475.

31 Bougenot MP, Tordi N, Betik AC, Martin X, Le Foll D, Parratte B et al. Effects of a wheelchair ergometer training programme on spinal cord-injured persons. Spinal Cord 2003; 41: 451-456.

32 Carvalho DCL, Garlipp CR, Bottini PV, Afaz SH, Moda MA, Cliquet Jr A. Effect of treadmill gait on bone markers and bone mineral density of quadriplegic subjects. Braz J Med Biol Res 2006; 39: 1357-1363.

33 Carvalho DC, Cliquet Jr A, Rondina JM, Cendes F. Electrical stimulation during gait promotes increase of muscle cross-sectional area in quadriplegics: a preliminary study. Clin Orthop Relat Res 2009; 467: 553-557.

34 Chen SC, Lai CH, Chan WP, Huang MH, Tsai HW, Chen JJJ. Increases in bone mineral density after functional electrical stimulation cycling exercises in spinal cord injured patients. Disabil Rehabil 2005; 27: 1337-1341.

35 Clark JM, Jelbart M, Rischbieth H, Strayer J, Chatterton B, Schultz C et al. Physiological effects of lower extremity functional electrical stimulation in early spinal cord injury: lack of efficacy to prevent bone loss. Spinal Cord 2007; 45 : 78-85.

36 Coupaud S, Jack LP, Hunt KJ, Allan DB. Muscle and bone adaptations after treadmill training in incomplete spinal cord injury: a case study using peripheral quantitative computed tomography. J Musculoskelet Neuronal Interact 2009; 9: 288-297.

37 Crameri RM, Weston A, Climstein M, Davis GM, Sutton JR. Effects of electrical stimulation-induced leg training on skeletal muscle adaptability in spinal cord injury. Scand J Med Sci Sports 2002; 12: 316-322.

38 de Groot PCE, Hjeltnes N, Heijboer AC, Stal W, Birkeland K. Effect of training intensity on physical capacity, lipid profile and insulin sensitivity in early rehabilitation of spina cord injured individuals. Spinal Cord 2003; 41: 673-679.

39 De Mello MT, Silva AC, Esteves AM, Tufik S. Reduction of periodic leg movement in individuals with paraplegia following aerobic physical exercise. Spinal Cord 2002; 40 646-649.

40 Dolbow DR, Miller J, Harnish C, Poarch H, Gorgey A, Gater DR. Arm crank exercise increases VO2 peak and reduces body fat mass in older adult with chronic paraplegia. Clin Kinesiology 2010; 64: 51-55.

41 Dyson-Hudson TA, Sisto SA, Bond Q, Emmons R, Kirshblum SC. Arm crank ergometry and shoulder pain in persons with spinal cord injury. Arch Phys Med Rehabil 2007; 88: 1727-1729.

42 Effing TW, Van Meeteren NLU, Van Asbeck FWA, Prevo AJH. Body weight-supported treadmill training in chronic incomplete spinal cord injury: a pilot study evaluating functional health status and quality of life. Spinal Cord 2006; 44: 287-296.

43 El-Sayed MS, Younesian A. Lipid profiles are influenced by arm cranking exercise and training in individuals with spinal cord injury. Spinal Cord 2005; 43: 299-305.

44 Gerrits HL, de Haan A, Sargeant AJ, van Langen H, Hopman MT. Peripheral vascula changes after electrically stimulated cycle training in people with spinal cord injury. Arch Phys Med Rehabil 2001; 82: 832-839.

45 Gerrits HL, Hopman MTE, Sargeant AJ, Jones DA, De Haan A. Effects of training on contractile properties of paralyzed quadriceps muscle. Muscle Nerve 2002; 25: 559-567.

46 Gerrits HL, Hopman MTE, Offringa C, Engelen BGM, Sargeant AJ, Jones DA et al. Variability in fibre properties in paralysed human quadriceps muscles and effects of training. Eur J Physiol 2003; 445: 734-740.

47 Giangregorio LM, Hicks AL, Webber CE, Phillips SM, Craven BC, Bugaresti JM et al. Body weight supported treadmill training in acute spinal cord injury: impact on muscle and bone. Spinal Cord 2005; 43: 649-657.

48 Giangregorio LM, Webber CE, Phillips SM, Hicks AL, Craven BC, Bugaresti JM et al. Can body weight supported treadmill training increase bone mass and reverse muscle atrophy in individuals with chronic incomplete spinal cord injury? App/ Physiol Nutr Metab 2006; 31: 283-291.

49 Gorgey AS, Shepherd C. Skeletal muscle hypertrophy and decreased intramuscular fat after unilateral resistance training in spinal cord injury: case report. J Spinal Cord Med 2010; 33: 90-95.

50 Gregory CM, Bowden MG, Jayaraman A, Shah P, Behrman A, Kautz SA et al. Resistance training and locomotor recovery after incomplete spinal cord injury: a case series. Spinal Cord 2007; 45: 522-530.

51 Griffin L, Decker MJ, Hwang JY, Wang B, Kitchen K, Ding Z et al. Functional electrical stimulation cycling improves body composition, metabolic and neural factors in persons with spinal cord injury. J Electromyography Kinesiol 2009; 19: 614-622.

52 Grigorenko A, Bjerkefors A, Rosdahl H, Hultling C, Alm M, Thorstensson A. Sitting balance and effects of kayak training in paraplegics. J Rehabil Med 2004; 36: 110-116.

53 Harvey LA, Fornusek C, Bowden JL, Pontifex N, Glinsky J, Middleton JW et al. Electrical stimulation plus progressive resistance training for leg strength in spinal cord injury: a randomized controlled trial. Spinal Cord 2010; 48: 570-575.

54 Heesterbeek PJC, Berkelmans HWA, Thijssen DHJ, van Kuppevelt HJM, Hopman MTE, Duysens J. Increased physical fitness after 4-week training on a new hybrid FES-cycle in persons with spinal cord injury. Technol Disabil 2005; 17: 103-110.

55 Hicks AL, Martin KA, Ditor DS, Latimer AE, Craven C, Bugaresti J et al. Long-term exercise training in persons with spinal cord injury: Effects on strength, arm ergometry performance and psychological well-being. Spinal Cord 2003; 41: 34-43.

56 Hicks AL, Adams MM, Martin Ginis K, Giangregorio L, Latimer A, Phillips SM et al. Long-term body-weight-supported treadmill training and subsequent follow-up in 
persons with chronic $\mathrm{SCl}$ : effects on functional walking ability and measures of subjective well-being. Spinal Cord 2005; 43: 291-298.

57 Jacobs PL, Nash MS, Rusinowski Jr JW. Circuit training provides cardiorespiratory and strength benefits in persons with paraplegia. Med Sci Sports Exerc 2001; 33: $711-717$.

58 Jacobs PL. Effects of resistance and endurance training in persons with paraplegia. Medicine and Science in Sports and Exercise 2009; 41: 992-997.

59 Jeon JY, Weiss CB, Steadward RD, Ryan E, Burnham RS, Bell G et al. Improved glucose tolerance and insulin sensitivity after electrical stimulation-assisted cycling in people with spinal cord injury. Spinal Cord 2002; 40: 110-117.

60 Jeon JY, Hettinga D, Steadward RD, Wheeler GD, Bell G, Harber V. Reduced plasma glucose and leptin after 12 weeks of functional electrical stimulation rowing exercise training in spinal cord injury patients. Arch Phys Med Rehabil 2010; 91: 1957-1959.

61 Kakebeeke TH, Hofer PJ, Frotzler A, Lechner HE, Hunt KJ, Perret C. Training and detraining of a tetraplegic subject: High-volume FES cycle training. Am J Phys Med Rehabil 2008; 87: 56-64.

62 Kern H, Carraro U, Adami N, Hofer C, Loefler S, Vogelauer M et al. One year of homebased daily FES in complete lower motor neuron paraplegia: recovery of etanic contractility drives the structural improvements of denervated muscle. Neurol Res 2010; 32: 5-12.

63 Kjær M, Mohr T, Biering-Sørensen F, Bangsbo J. Muscle enzyme adaptation to training and tapering-off in spinal-cord-injured humans. Eur J Appl Physiol 2001; 84: $482-486$.

64 Latimer AE, Martin Ginis KA, Hicks AL, McCartney N. An examination of the mechanisms of exercise-induced change in psychological well-being among people with spinal cord injury. J Rehabil Res Dev 2004; 41: 643-651.

65 Latimer AE, Martin-Ginis KA, Hicks AL. Buffering the effects of stress on well-being among individuals with spinal cord injury: a potential role for exercise. Ther Recreation J 2005; 39: 131-138.

66 Liu CW, Chen SC, Chen CH, Chen TW, Chen JJJ, Lin CS et al. Effects of functional electrical stimulation on peak torque and body composition in patients with incomplete spinal cord injury. Kaohsiung J Med Sci 2007; 23: 232-240

67 Mahoney ET, Bickel CS, Elder C, Black C, Slade JM, Apple Jr D et al. Changes in skeletal muscle size and glucose tolerance with electrically stimulated resistance training in subjects with chronic spinal cord injury. Arch Phys Med Rehabil 2005; 86 : 1502-1504.

68 Martin-Ginis KA, Latimer AE, McKechnie K, Ditor DS, McCartney N, Hicks AL et al. Using exercise to enhance subjective well-being among people with spinal cord injury: the mediating influences of stress and pain. Rehabil Psychol 2003; 48: 157-164.

69 Mohr T, Dela F, Handberg A, Biering-Sorensen F, Galbo H, Kjaer M. Insulin action and long-term electrically induced training in individuals with spinal cord injuries. Med Sci Sports Exerc 2001; 33: 1247-1252.

70 Nash MS, van Elk N, Johnson BM. Effects of circuit resistance training on fitness attributes and upper-extremity pain in middle-aged men with paraplegia. Arch Phys Med Rehabil 2007; 88: 70-75.

71 Phillips SM, Stewart BG, Mahoney DJ, Hicks AL, McCartney N, Tang JE et al. Bodyweight-support treadmill training improves blood glucose regulation in persons with incomplete spinal cord injury. J Appl Physiol 2004; 97: 716-724.

72 Skold C, Lonn L, Harms-Ringdahl K, Hulting C, Levi R, Nash M et al. Effects of functional electrical stimulation training for six months on body composition and spasticity in motor complete tetraplegic spinal cord-injured individuals. J Rehabil Med 2002; 35: 25-32.

73 Soyupek F, Savas S, Ozturk O, Ilgun E, Bircan A, Akkaya A. Effects of body weight supported treadmill training on cardiac and pulmonary functions in the patients with incomplete spinal cord injury. J Back Musculoskelet Rehabil 2009; 22: 213-218.

74 Stewart BG, Tarnopolsky MA, Hicks AL, McCartney N, Mahoney DJ, Staron R et al. Treadmill training-induced adaptations in muscle phenotype in persons with incomplete spinal cord injury. Muscle Nerve 2004; 30: 61-68.

75 Stoner L, Sabatier MJ, Mahoney ET, Dudley GA, McCully KK. Electrical stimulationevoked resistance exercise therapy improves arterial health after chronic spinal cord injury. Spinal Cord 2007; 45: 49-56.

76 Sutbeyaz ST, Koseoglu BF, Gokkaya NK. The combined effects of controlled breathing techniques and ventilatory and upper extremity muscle exercise on cardiopulmonary responses in patients with spinal cord injury. Int J Rehab Res 2005; 28: 273-276.

77 Thijssen DH, Heesterbeek P, van Kuppevelt DJ, Duysens J, Hopman MT. Local vascula adaptations after hybrid training in spinal cord-injured subjects. Med Sci Sports Exerc 2005; 37: 1112-1118.

78 Valent LJ, Dallmeijer AJ, Houdijk H, Slootman HJ, Janssen TW, Post MW et al. Effects of hand cycle training on physical capacity in individuals with tetraplegia: a clinical trial. Phys Ther 2009; 89: 1051-1060.

79 Valent L, Dallmeijer A, Houdijk H, Slootman HJ, Janssen TW, Van Der Woude LH. Effects of hand cycle training on wheelchair capacity during clinical rehabilitation in persons with a spinal cord injury. Disabil Rehabil 2010; 32: 2191-2200.

80 Van Duijnhoven N, Hesse E, Janssen T, Wodzig W, Scheffer P, Hopman M. Impact of exercise training on oxidative stress in individuals with a spinal cord injury. Eur J Appl Physiol 2010; 109: 1059-1066.

81 Wheeler GD, Andrews B, Lederer R, Davoodi R, Natho K, Weiss C et al. Functional electric stimulation-assisted rowing: increasing cardiovascular fitness through functional electric stimulation rowing training in persons with spinal cord injury. Arch Phys Med Rehabil 2002; 83: 1093-1099.

82 Hassett L, Moseley AM, Tate R, Harmer AR. Fitness training for cardiorespiratory conditioning after traumatic brain injury. Cochrane Database Syst Rev 2008, CD006123.

83 Voet N, Van der Kooi EL, Riphagen I, Lindeman E, van Engelen B, Geurts A. Strength training and aerobic exercise training for muscle disease. Cochrane Database Syst Rev 2010, CD003907.

84 Masala C, Petretto DR. From disablement to enablement: conceptual models of disability in the 20th century. Disabil Rehabil 2008; 30: 1233-1244.

85 Mortenson WB, Miller WC, Auger C. Issues related to the selection of wheelchairspecific activity and participation outcome measures: A review. Arch Phys Med Rehabil 2008; 89: 1177-1186.

86 Noonan VK, Miller WC, Loreau L. A review of instruments assessing participation in persons with spinal cord injury. Spinal Cord 2009; 47: 435-446.

87 Jacobs PL, Nash MS. Exercise recommendations for individuals with spinal cord injury. Sport Med 2004; 34: 727-751.

88 Nash MS. Exercise as a health-promoting activity following spinal cord injury. J Neurol Phys Ther 2005; 29: 87-103, 106

89 Noreau L, Shephard RJ. Spinal cord injury, exercise and quality of life. Sport Med 1995; $20: 4$ 226-50. 